\title{
Socios o clientes: educación para la participación ciudadana en el Tercer Sector
}

\section{Partners or Clients: Education for Citizen Participation in the Third Sector}

\author{
Fran J. Garcia-Garcia ${ }^{1}$ \\ garfran6@uv.es \\ CRUZ PÉREZ-PÉREZ \\ cruz.perez@uv.es \\ Universidad de Valencia, España
}

\section{Resumen:}

La participación ciudadana es clave para desarrollar sociedades democráticas y garantizar una educación para todos. En este sentido, el Tercer Sector de acción social y educativa es un escenario imprescindible. Sin embargo, existen problemas en su funcionamiento procedentes de la aplicación de evaluaciones externas sobre la excelencia y calidad de los servicios. Dichos problemas han sido analizados para definir el estado actual del Tercer Sector y sus implicaciones educativas, ofreciendo soluciones y orientaciones para la práctica. Se ha abordado el reparto de responsabilidades para el bienestar, la influencia de la evaluación externa en el Tercer Sector, las carencias de capacidad ciudadana para participar en el mismo, y los retos y funciones de la educación en este marco. El resultado del análisis sugiere proteger el sentido original del Tercer Sector, descentralizar la influencia de las evaluaciones externas y aprovechar las tendencias pedagógicas de los últimos tiempos. Además, se concluye en la necesidad de una alianza entre la educación formal y el Tercer

\begin{abstract}
:
Citizen participation is a key to develop democratic societies which guarantee education for all. In this sense, the Third Sector for social and educational action is an essential scenario. However, there are operational problems arising from the application of external evaluations on service excellence and quality. These problems are analysed to define the current state of the Third Sector and its educational implications, offering solutions and guidance for practice. Distribution of responsibilities for welfare, influence of external evaluation on the Third Sector, lack of citizen ability to participate in it, and challenges and functions of education within this framework are addressed. The results of the analysis highlight protecting the original meaning of the Third Sector, decentralising the influence of external evaluations and taking advantage of recent pedagogical trends. In addition, the conclusion points to the need for an alliance between formal education and the Third Sector to train empathic and morally autonomous citizens.
\end{abstract}

1 Dirección para correspondencia (correspondence address):

Fran J. García García. Departamento de Teoría de la Educación. Universidad de Valencia. Campus de Blasco Ibáñez. Av. Blasco Ibáñez, 30. 46010, València (España). 
Socios o clientes: educación para la participación ciudadana en el Tercer Sector

Sector para formar ciudadanos empáticos y moralmente autónomos.

\section{Palabras clave:}

Participación; ciudadanía; tercer sector; educación inclusiva; educación en valores.

\section{Key words:}

Participation; citizenship; third sector; inclusive education; values education.

\section{Résumé :}

La participation des citoyens est essentielle au développement des sociétés démocratiques et pour garantir l'éducation pour tous. En ce sens, le troisième secteur d'action sociale et éducative est un scénario fondamental. Cependant, il y a des problèmes dans son fonctionnement à cause de l'application des évaluations externes sur l'excellence et la qualité des services. Ces problèmes ont été analysés pour définir le statut actuel du tiers secteur et ses implications pédagogiques comme des solutions et des lignes directrices pour la pratique. La répartition des responsabilités pour le bien-être, l'influence de l'évaluation externe dans le tiers secteur, le pénurie de capacité des citoyens à y participer, et les défis et les fonctions de l'éducation ont été abordés dans ce cadre. Le résultat de l'analyse suggère de protéger le sens originel du tiers secteur, décentraliser l'influence des évaluations externes et profiter des tendances pédagogiques actuelles. En outre, l'étude conclut avec la nécessité d'une alliance entre l'éducation formelle et le tiers secteur dans la formation des citoyens empathiques et moralement autonomes.

\section{Mots clés:}

Participation; citoyenneté; tiers secteur; éducation inclusive; éducation en valeurs.

Fecha de recepción: 1-6-2018

Fecha de aceptación: 15-9-2018

\section{Introducción}

La educación es un valor que se aprende y genera condiciones educativas, no solo en la escuela, sino también en las organizaciones del Tercer Sector (TS). El auge de este último es cada vez más notable en la configuración de las sociedades actuales, seguramente por ofrecer una respuesta a las necesidades socioeducativas desde entidades sin ánimo de lucro, aunque asuma menor representación institucional que el resto de organismos que se dedican a este fin. Enlazando con el Objetivo de Desarrollo Sostenible 4 de la UNESCO -una educación inclusiva y equitativa de calidad-, la participación ciudadana significa el acceso a los servicios socioeducativos de manera íntegra y coherente. Desde la paideia griega y la civitas romana el ser humano ha mantenido su preocupación por educarse para ello.

En la actualidad, la excelencia parece estar influyendo cada vez más en el funcionamiento del TS. La evaluación externa para la calidad de 
los servicios socioeducativos obedece a intereses y entendimientos distintos a los originales de las organizaciones no lucrativas. Lógicamente, las circunstancias están teñidas de paradojas y contrariedades que afectan seriamente al sentido de la participación ciudadana en varias direcciones. La cuestión es que existe una diferencia fundamental entre un TS con servicios para socios o para clientes: la capacidad de participación ciudadana.

El primer objetivo de este trabajo no es tanto renunciar a la evaluación externa, sino más bien plantear sus inconvenientes, carencias y contradicciones para mejorarla. El segundo es analizar las implicaciones del actual estado del TS para una educación focalizada en la participación ciudadana. Para esto, el trabajo se desarrolla entorno a diferentes ejes analíticos que van jalonando las implicaciones educativas. A lo largo del documento se ponen de relieve una reflexión crítica sobre el estado de la cuestión y el contraste de modelos para el funcionamiento del TS. La novedad radica en la adaptación de soluciones planteadas en otros momentos, para otros escenarios, al problema de la educación para la participación ciudadana sobre el TS.

\section{Los responsables del bienestar}

La educación es un derecho básico, reconocido durante setenta años en el artículo 26 de la Declaración Universal de los Derechos Humanos (ONU, 1948). Se trata, por tanto, de un elemento que proporciona bienestar a un determinado conjunto de ciudadanos. Asegurar su participación en contextos educativos es un factor clave para permitir el acceso global a la educación (Booth, y Ainscow, 2015). Es más, suprimir barreras de participación constituye un mecanismo efectivo para garantizar el derecho a la Educación para Todos, por lo que ha sido suscrito por una cantidad considerable de estados a lo largo de diferentes documentos normativos (UNESCO, 2016). Pero la participación es, asimismo, un elemento sustancial del ejercicio ciudadano por encima de la clásica Educación Cívica y hacia el desarrollo de perspectivas más complejas. La formación de ciudadanos va en la línea de la autonomía moral kantiana y las capacidades empáticas (Pérez-Pérez, 2016). Así lo asumen también las últimas tendencias pedagógicas como los proyectos de AprendizajeServicio, y no únicamente en el marco de la escuela, sino con la estrecha 
colaboración de las organizaciones del TS (Puig, Batlle, Bosch, y Palos, 2006).

Los tres agentes encargados de proporcionar bienestar a los ciudadanos han sido la familia, el Estado y el mercado (Esping-Andersen, 1999). Desde la economía postindustrial, esta tríada ha ido forjando las tendencias en cada país hasta la llegada del estado relacional (Cooke, y Muir, 2012). Por supuesto, las diferentes regiones con cierta autonomía política continúan marcando las rutas para definir roles y repartir responsabilidades entre los mismos actores. Sin embargo, la reducción del intervencionismo estatal en cuestiones socioeducativas y el auge del TS de acción social dibujan un escenario diferente (Addicott, 2017). El bienestar sigue proporcionándose desde entidades públicas, pero también privadas con y sin ánimo de lucro. Evidentemente, las lógicas de funcionamiento son distintas en cada tipo de organización. En última instancia, se trata de un Welfare-Mix donde unos desean abarcar la máxima oferta de bienestar a cambio de un beneficio mientras otros sencillamente pretenden satisfacer sus necesidades asociándose o fundando plataformas sociales. En cualquiera de los casos, el énfasis está cada vez más en el individuo como responsable del bienestar (McCuaig, Enright, Rossi, Macdonald, y Hansen, 2017; Peters, 2016). Una de las más intensas implicaciones educativas que subyacen a la iniciativa privada es la diferencia entre los servicios socioeducativos con participación ciudadana y los que están basados en modelos de calidad que no necesariamente asumen dicha participación. Para determinar esta analogía conviene prestar atención a la evaluación externa en las organizaciones del TS.

\section{Influencia de la evaluación externa}

Las organizaciones del TS colaboran formando redes con otras entidades, públicas o privadas, para ganar capacidad institucional cuando la oferta de servicios persigue fines similares (Iglesias, y Carreras, 2013). Es un modo de destinar recursos para conseguir resultados independientemente de los procesos. Más aún, los motivos que guían estas alianzas estratégicas tienen poco o nada que ver con la participación de socios o ciudadanos en los procesos de acción socioeducativa (Figura 1). 


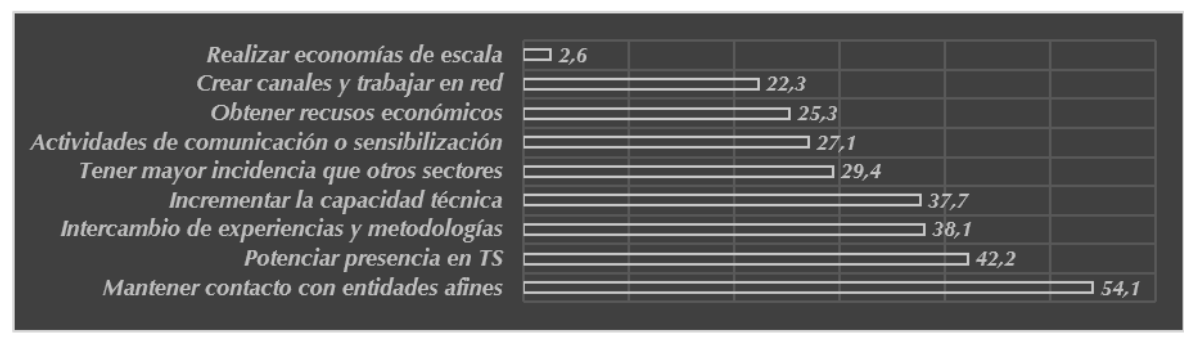

Figura 1. Motivos para la adhesión a otras entidades en organizaciones del TS. Copyright 2015 por Systeme Innovación y Consultoría.

En este contexto, se articulan varios mecanismos de evaluación y certificación de los servicios que ofrece el TS. Dos de los más destacados son el modelo de la European Foundation for Quality Management (EFQM) y las normas de la International Organization for Standardization (ISO). Tampoco estos suman la participación ciudadana en los procesos de acción socioeducativa entre sus orientaciones (Tabla 1).

Tabla 1

Líneas de acción del modelo EFQM y las normas ISO

\begin{tabular}{cc}
\hline EFQM & $I S O$ \\
\hline Ofrecer valor añadido al consumidor & Responder a una necesidad de mercado \\
Crear un futuro sostenible & Atender la opinión de expertos mundiales \\
Desarrollar capacidad organizativa & Atender a todas las partes interesadas \\
Crear innovación y creatividad & Atender a consensos \\
Liderar con visión, inspiración e & \\
integridad & \\
Gestionar con agilidad & \\
Mejorar con el talento de las personas & \\
Mantener resultados excepcionales &
\end{tabular}

Fuentes: EFQM (2017) e ISO (2017).

Aunque se trata de enfoques considerablemente centrados en el mercado, parece que los progresos que aportan sobre los resultados o los beneficios económicos no acaban de aclararse. Pese a ello, se han detectado mejoras en la calidad de sus procesos (Melão, Guia, y Amorim, 2016), que no implican en esencia la participación ciudadana. Resta decir que la opinión de expertos mundiales que persigue la ISO puede 
entrar en conflicto con la búsqueda de consensos y la resolución de necesidades socioeducativas locales. En cuanto al mantenimiento de resultados excepcionales que pretende la EFQM, sin financiación suficiente resulta inviable y ata al TS a las dinámicas de productividad mercantil para sobrevivir ante la reducción del gasto público.

Con todo, una financiación externa supone una dependencia externa. A parte del 55.3\% de financiación pública al TS registrada en 2013, el $19.4 \%$ provino de entidades privadas y el $25.3 \%$ por cuenta propia (Systeme Innovación y Consultoría, 2015). Casi la mitad de los fondos privados podrían implicar una influencia externa mediante rendición de cuentas al mercado. En consecuencia, evaluar conforme a parámetros no compartidos o que no responden al sentido original de las organizaciones conlleva cambios de funcionamiento y la pérdida del sentido original. Así es como se reduce la preocupación por que los ciudadanos se conviertan en meros receptores pasivos acreditando los servicios del TS con sellos de calidad.

\section{Una ciudadanía activa y desposeída}

Las organizaciones del TS están formadas por personas o entidades unidas a fin de satisfacer necesidades socioeducativas. En la medida en que necesitan conseguir acreditaciones de calidad para rendir cuentas al sector público o a empresas privadas pasan a ser parcialmente absorbidas. En el primer caso, se privatiza la oferta de servicios públicos; en el segundo, se pone el capital social al servicio de intereses particulares. De cualquier forma, las lógicas originales del TS son diferentes a las de los otros agentes e implican usos y funcionamientos distintos (Tabla 2). De cara al sector público, las problemáticas comunes que dan sentido al TS no necesariamente se consideran de interés general y susceptibles de merecer inversión pública. De cara al mercado, lejos de producir bienes de consumo, las organizaciones del TS tratan de satisfacer las necesidades locales cuando la oferta de bienestar que otros órganos proporcionan no llega a todos. Desde luego, fomentar el activismo desde centros socioeducativos mientras se cercena y desarraiga el control y la participación ciudadana de las propias entidades no parece tener mucho sentido.

De algún modo, este panorama recuerda al opting out en la escuela. 
Cuando se publicaron informes como $A$ Nation at Risk el mes de abril de 1983, en los Estados Unidos, o Better School en marzo de 1985, en Reino Unido, quedó en evidencia el empobrecimiento de la calidad escolar. La solución de los gobiernos neoliberales y neoconservadores consistió en defender la libertad de los padres y madres a elegir centro docente otorgándoles el derecho a sacar a sus hijos de un colegio para matricularlos en otro (Carl, 1994). Sin embargo, seleccionar la mejor inversión en términos económicos no requiere la participación del consumidor en la gestión y proceso que da lugar a los bienes. Ya no es que la participación ciudadana sea indispensable para lograr sociedades realmente democráticas, es que son las propias barreras que coartan la participación las que impiden el acceso a una educación real y de calidad.

Mientras el ordenamiento de una institución se sustenta sobre la obligatoriedad, el de una organización de carácter voluntario no (Weber, 2000). A diferencia de la institución escolar, el TS necesita la implicación ciudadana para seguir existiendo. En la actualidad se publican informes que advierten de la calidad con que el TS responde ante una crisis (uno de los últimos: Systeme Innovación y Consultoría, 2015). La cuestión es si será posible incluir la participación ciudadana entre los parámetros de calidad para dar paso a la mutua construcción de realidades socioeducativas. Freire (2012) defendió que nadie educa a nadie, sino que todos nos educamos en relación y mediatizados por un contexto donde coexisten diferentes discursos. Pero lo cierto es que ya hay quienes consideran difícil organizarse sin organizaciones (Polanska, y Chimiak, 2016).

Tabla 2

Diferencias entre las perspectivas del TS y de las empresas evaluadoras

\begin{tabular}{lcc}
\hline & TS & Empresas evaluadoras \\
\hline El ciudadano & Participante/Socio & Consumidor/Cliente \\
Los educadores & Profesionales & Productor/Plantilla \\
El TS & Espacio común & Empresa/Almacén \\
La acción educativa & Satisfacción de necesidades & Producto/Bienes \\
\hline
\end{tabular}

Fuente: elaboración propia 


\section{El trayecto intermedio de la educación}

La educación es reconocida mundialmente, no solo como un derecho humano, sino como un bien público (Daviert, 2016). En este sentido, la educación es un fin en sí misma (UNESCO, 2015) y constituye tanto un proceso como un producto. El proceso tiene que ver con la participación en la gestión y toma de decisiones, y el producto con los resultados que se consiguen a lo largo del tiempo. Si la gestión y toma de decisiones vienen marcadas por estándares de organismos con lógicas ajenas al TS -como el modelo EFQM o las normas ISO-, la ciudadanía queda desarraigada de los procesos socioeducativos. Estos se transforman en pura formación de personal para cumplir aquellos estándares: formación para voluntarios, para estudiantes en prácticas e incluso para los propios profesionales contratados. El fin principal es obtener un sello que acredite la calidad de la organización más que la generación de fuentes para la educación en valores de convivencia y participación ciudadana.

Efectivamente, la educación como proceso entraña fines. Además, sin formación técnica no es posible satisfacer las necesidades por las cuales las personas se organizan en el TS. Por tanto, es importante la existencia de procesos formativos que generen convivencia en la resolución de necesidades, dando paso a procesos educativos en sentido freireano. Por otro lado, recientemente se han realizado estudios examinando la asociación entre el capital social y la participación voluntaria. Uno de los últimos concluye que el nivel de estudios y la educación a lo largo de toda la vida están directamente relacionados con las conductas participativas (Vera-Toscano, Rodrigues, y Costa, 2017). Las evidencias ponen de relieve la necesidad de considerar seriamente la educación como un fin y no solo como un medio. Por lo demás, convendría tener presente las diferencias entre instrucción y educación.

Parece que el panorama requiere una red entre el TS y una escuela que enseñe para la participación ciudadana, con énfasis en un currículum que recoja la conciencia empática y la autonomía moral. Es evidente que el ejercicio activo de la ciudadanía pasa por institucionalizar diversas vías de formación en estrecha relación con el TS. De otro modo, difícilmente se asimilarán las competencias cívicas adecuadas. 


\section{Retos educativos para la participación ciudadana}

Un primer reto consiste en formar ciudadanos competentes para gozar de los derechos sociales, civiles y políticos de su comunidad, capaces de participar en las decisiones económicas que afectan a sus vidas, con un compromiso altruista a nivel local y global (Escámez, y Gil, 2002). Sobre todo, cuando resulta difícil situar la soberanía, la autonomía y la accountability entre las zonas locales y supranacionales (Howard, 1981), y llegados a un punto en que el cambio a corto plazo se ha convertido en un imperativo social, que los sistemas educativos deben afrontar con ciertas garantías de éxito (Säfström, 2018). Los miembros de una comunidad comparten rasgos identificadores que los diferencian de los demás (Cortina, y Conill, 2001). En el caso de las organizaciones del TS, son las particularidades las que generan unión. Por tanto, aunque existan modelos de evaluación generales, conviene prestar atención a las singularidades de una comunidad que se organiza.

La libertad es una cualidad fundamental para las relaciones humanas en la sociedad civil, al igual que es reivindicada por el mercado para las relaciones comerciales. En esta línea, la libertad suscita una analogía entre los intereses individuales y el bienestar general (Giddens, 1999), lo que sitúa la participación en una escala de tonos grises.

Por una parte, está la libertad como no-dominación (Pettit, 1997), en atención a la cual una comunidad es libre en tanto que no hay interferencia de agentes poderosos como los organismos estatales. Ahora bien, las dependencias económicas del TS sobre la esfera estatal y el mercado esbozarían un reto considerable hacia la no-dominación que conduce a preguntarse por nuevas vías de financiación, si las hay. Por otra parte, la libertad como capacidad (Sen, 1999) contempla las oportunidades de la ciudadanía para actuar, decidir e influir en la comunidad. Ahora bien, no únicamente es necesaria una formación expresa para ello, sino que la dominación del TS por parte de las lógicas de mercado merma la capacidad de autogestión, propia de una ciudadanía democrática. Incluso se ha señalado la asociación humana como condición para adquirir el poder de influir y cambiar los sucesos sociales y políticos (Arendt, 1998).

Por añadidura, la pedagogía pragmática de Dewey (2002) invita a la deliberación común a fin de conocer las posibles actuaciones e implicaciones para la práctica social y educativa. Este enfoque conlleva un tipo de interacción popular sugerente para aprender con y de los otros. No 
obstante, la deliberación requiere un margen de libre participación con capacidad de influir sobre las consecuencias prácticas que tienen lugar en la comunidad. En este punto, parece que existe un conflicto con las lógicas de mercado, contrarias al carácter no-lucrativo de la participación ciudadana en el TS. Otro reto sería, por tanto, el desarrollo de acciones socioeducativas en atención a un modelo de justicia social como el de Nussbaum (2009) y no solo hacia los modelos de excelencia. Ello supone centrar la responsabilidad del bienestar en los poderes públicos y no tanto en las personas afectadas por necesidades no resueltas. Junto con esto, hay que recordar que la supresión de barreras de participación resulta imprescindible para garantizar una Educación para Todos.

\section{Conclusiones: socios o clientes}

Existen diferencias evidentes entre los servicios socioeducativos con y sin participación ciudadana real. También las hay entre formación para cumplir estándares y formación como fuente de educación en valores de convivencia y participación ciudadana, que den respuesta a las necesidades de las personas organizadas en el TS. Ante las contradicciones de los modelos de excelencia, una solución posible consiste en descentralizar el margen de influencia sobre la gestión de servicios y asumir que las lógicas de funcionamiento son diferentes para las organizaciones privadas con y sin ánimo de lucro. Además, la financiación externa reduce la preocupación por una ciudadanía pasiva, acentuando la tendencia hacia la acreditación de la calidad. En este marco, parece ser cada vez más necesaria una alianza escuela-TS hacia la participación ciudadana, con un currículum que incorpore la conciencia empática y la autonomía moral. Aunque existan modelos de evaluación generales, conviene prestar atención a las singularidades de cada comunidad del TS. No porque la evaluación externa sea negativa, que de hecho es necesaria para mejorar, sino porque los planteamientos vigentes no resultan coherentes con el sentido del TS y la participación ciudadana.

Si las organizaciones del TS están formadas por socios podrán recuperar parte de la autonomía y sentido que les es propio, mientras se lo puedan costear con la misma autonomía y sentido. Si se trata de clientes, deberán abandonar esto para convertirse en un grupo de consumidores pasivos, con una pequeña élite a la cabeza que se preocupe por rendir 
cuentas a organismos externos con intereses muy distintos. La participación ciudadana está en la cuerda floja y depende de que los modelos de evaluación de la excelencia introduzcan en sus planteamientos el valor de la educación como fin en sí misma y la necesidad de recobrar el significado original de las organizaciones del TS mediante la participación de sus miembros en la acción socioeducativa. Mientras tanto, no faltan quienes se cuestionan si el TS no es una senda de austeridad hacia la privatización de la oferta pública (Myers, 2017).

Los nuevos avances en educación ofrecen constantemente soluciones potenciales como el Aprendizaje-Servicio, los servicios a la comunidad, las comunidades de aprendizaje, etcétera. Sin embargo, hace falta institucionalizar, financiar, formar, regular, estudiar e investigar para seguir avanzando, operativizando las propuestas y hallando estándares comunes para evaluar hasta qué punto son útiles para impulsar la participación ciudadana. El valor añadido del capital social que tiene su foco en el TS es, sin duda, la participación ciudadana: una fuente de educación en valores para aprender a convivir y, como apuntan los modelos de excelencia, mejorar continuamente, pero en calidad de personas.

\section{Referencias}

Addicott, K. (2017). There may be trouble ahead: exploring the changing shape of nonprofit entrepreneurship in third sector organizations. Public Money \& Management, 37(2), 81-88, doi:10.1080/09540962.2016.1266142

Arendt, H. (1998). The Human Condition. Chicago, IL: University of Chicago Press.

Booth, T. y Ainscow, M. (2015). Guía para la Educación Inclusiva. Desarrollando el aprendizaje y la participación en los centros escolares. Madrid: OEI y FUHEM.

Carl, J. (1994). Parental Choice as National Policy in England and the United States. Comparative Education Review, 38(3), 294-322. doi:10.1086/447254

Cooke, G. y Muir, R. (Eds.). (2012). The relational state. How recognising the importance of human relationships could revolutionise the role of the state. London: Institute for Public Policy Research.

Cortina, A. y Conill, J. (2001). Educar en la ciudadanía. Valencia: Instituto Alfonso el Magnánimo.

Daviet, B. (2016). Revisiting the Principle of Education as a Public Good [Sitio web]. Recuperado de http://unesdoc.unesco.org/images/0024/002453/245306E.pdf

Dewey, J. (2002). Human Nature and Conduct. North Chelmsford, MA: Courier Corporation.

EFQM. (2017). EFQM Model. Fundamental concepts [Sitio web]. Recuperado de http:// www.efqm.org/efqm-model/fundamental-concepts 
Socios o clientes: educación para la participación ciudadana en el Tercer Sector Fran J. Garcia-Garcia y Cruz pérez-pérez

Escámez, J. y Gil, R. (2002). La educación de la ciudadanía. Madrid: CCS-ICCE.

Esping-Andersen, G. (1999). Social Foundations of Postindustrial Economies. New York, NY: Oxford University Press.

Freire, P. (2012). Pedagogía del oprimido. Madrid: Siglo XXI.

Giddens, A. (1999). The Third Way: The Renewal of Social Democracy. Hoboken, NJ: Wiley.

Howard, M. (1981). War and the liberal conscience. Oxford: Oxford University Press.

Iglesias, M. y Carreras, I. (2013). La colaboración efectiva en las ONG. Alianzas estratégicas y redes. Barcelona: Instituto de Innovación Social ESADE y Fundación PwC.

ISO. (2017). How we develope standards [Sitio web]. Recuperado de https://www.iso. org/developing-standards.html

McCuaig, L., Enright, E., Rossi, A., Macdonald, D. y Hansen, S. (2016). An Eroding Social Justice Agenda: The Case of Physical Education and Health Edu-Business in Schools. Research Quarterly for Exercise and Sport, 87(2), 151-164. doi:10.1080/02701367. 2016.1163978

Melão, N. F., Guia, S. M. y Amorim, M. (2016). Quality Management and Excellence in the third sector: examining European Quality in Social Services (EQUASS) in nonprofit social services. Total Quality Management \& Business Excellence, 28(7/8), 840-857. doi:10.1080/14783363.2015.1132160

Myers, J. (2017). To austerity and beyond! Third sector innovation or creeping privatization of public sector services? Public Money \& Management, 37(2), 97-104. doi:10.1 080/09540962.2016.1266152

Nussbaum, M. C. (2009). Frontiers of Justice: Disability, Nationality, Species Membership. London: Harvard University Press.

ONU. (1948). Universal Declaration of Human Rights [Sitio web]. Recuperado de http:// www.un.org/en/universal-declaration-human-rights/

Pérez-Pérez, C. (2016). Educación en valores para la ciudadanía. Estrategias y técnicas de aprendizaje. Bilbao: Editorial Desclée de Brower, S.A.

Peters, M. A. (2016). From State responsibility for education and welfare to self-responsibilisation in the market. Discourse: Studies in the Cultural Politics of Education, 38(1), 138-145. doi:10.1080/01596306.2016.116385

Pettit, P. (1997). Republicanism: A Theory of Freedom and Government. New York, NY: Oxford University Press.

Polanska, D. V. y Chimiak, G. (2016). Organizing without organizations: on informal social activism in Poland. International Journal of Sociology and Social Policy, 36(9/10), 662-679. doi:10.1108/IJSSP-11-2015-0120

Puig, J. M., Batlle, R., Bosch, C., y Palos, J. (2006). Aprenentatge Servei. Educar per a la cuitadania. Barcelona: Ediciones Octaedro S.L.

Sen, A. (1999). Development as freedom. New York, NY: Anchor Books.

Säfström, C. A. (2018). Liveable life, educational theory and the imperative of constant change. European Educational Research Journal, 17(5), 621-630. doi:10.1177/1474904118784480

Systeme Innovación y Consultoría. (2015). El Tercer Sector de acción social. Impacto de la crisis. Madrid: Systeme Innovación y Consultoría. 
UNESCO. (2015). Rethinking Education. Towards a global common good? Paris: United Nations Educational, Scientific and Cultural Organization.

UNESCO. (2016). Implementing the Right to Education. A Compendium of practical examples. Paris: United Nations Educational, Scientific and Cultural Organization.

Vera-Toscano, E., Rodrigues M., y Costa, P. (2017). Beyond educational attainment: The importance of skills and lifelong learning for social outcomes. Evidence for Europe from PIAAC. European Journal of Education, 52(2), 217-231. doi:10.1111/ ejed.12211

Weber, M. (2000). Basic Concepts in Sociology. New York, NY: The Citadel Press. 
